\title{
Analysis of Ionospheric foF2 by Solar Activity over the Korean Peninsula
}

\author{
Min-Ho Jeon, Chang-Heon Oh \\ Department of Electrical, Electronics and Communication Engineering, Korea University of Technology and Education, \\ Choongnam, Korea
}

\begin{tabular}{l} 
Article Info \\
\hline Article history: \\
Received Aug 2, 2015 \\
Revised Nov 5, 2015 \\
Accepted Nov 22, 2015 \\
\hline
\end{tabular}

Keyword:

Critical frequency

F2 layer

Ionosphere

Shortwave communications

Solar activity

\begin{abstract}
The F2 layer is the upper sector of the ionospheric F region, and it is 250 $\mathrm{km}$ above sea level. It has a high electron density and thus plays an important role in shortwave communications. The variations of the critical frequency of the F2 layer (foF2) offer clues regarding the events happening within the entire F2 layer, and foF2 analysis is essential for stable shortwave communications. This study analyzes the seasonal and annual variations of the foF2 as well as the reactions of the F2 layer height at two locations in South Korea by employing the mean and standard deviation (SD) used in previous studies. To ensure a more elaborate analysis, the median and quartiles were used for analyzing the ionosphere. We thereby compensate for the limitations of the mean and SD in developing the SD, despite the convenience of the SD for probability analysis. The application of the median and quartiles for the analysis of ionospheric data led to analysis results with greater detail. This was achieved by determining the relative SD and concurrently displaying the outliers and range of variations.
\end{abstract}

Copyright (C) 2016 Institute of Advanced Engineering and Science. All rights reserved.

\section{Corresponding Author:}

Chang-Heon Oh,

Department of Electrical, Electronics and Communication Engineering

Korea University of Technology and Education,

Gajeon-Ri 307, Byungchun-myun, Chonan-Si, Choongnam, 330-708, Korea.

Email: choh@koreatech.ac.kr

\section{INTRODUCTION}

The F2 layer is the upper sector of the ionospheric F region. It is $\sim 250 \mathrm{~km}$ above sea level and plays an important role in shortwave communications because it has the highest electron density in the ionosphere. The variations in the critical frequency (foF2) of the F2 layer indicate the events occurring there. Ionospheric variations can be divided into four types: diurnal, seasonal, latitudinal, and solar-cyclic. The foF2 above the Korean Peninsula is the maximum at dawn and minimum at dusk, and the times for the peak and lowest point are subject to seasonal variation and solar-activity cycles [1], [2]. Research is conducted across the world to better understand such foF2 variations.

In this paper, seasonal and annual variations in the foF2 and height of the peak density (hmF2) are examined at two locations in Korea (Rep.)—Jeju-do and Icheon-si-during different solar-activity periods using the mean and standard deviation (SD). In addition to the mean and SD, the median and quartiles are used to explain the variations in the foF2. In previous studies, the mean and SD were useful to indicate the probability, but there were limitations in developing the SD. In the present study, by using the median and quartiles, which facilitate SD calculation, the range of variations in the foF2 due to solar activities is demonstrated in greater detail [3]. 


\section{DATA AND METHOD OF ANALYSIS}

The data used in this study are the hourly peaks of foF2 and hmF2 in Korea at two locations: Jeju-do (lat.: $33.43^{\circ} \mathrm{N}$; lon.: $126.3^{\circ} \mathrm{E}$; elev.: $68.0 \mathrm{~m}$ ) and Icheon-si (lat.: $37.14^{\circ} \mathrm{N}$; lon.: $127.54^{\circ} \mathrm{E}$; elev.: $68.0 \mathrm{~m}$ ). Raw data provided by the Korea Space Weather Center (http://www.spaceweather.go.kr/) were used, and the analysis results were verified using the International Reference Ionospheric model (IRI-2012). The ionospheric variations have turning points during the summer and winter solstices and spring and fall equinoxes [4], [5]. Therefore, we divided the raw data into four analysis datasets according to Korea's seasonal characteristics: spring (March-May), summer (June-August), fall (September-November), and winter (December-February). Additionally, the data were arranged in line with the solar-activity cycles in order to analyze the ionospheric variations according to the solar activity. Thus, we generated different datasets for solar activity: the low-solar-activity (LSA) dataset using the 2009 and 2010 data, medium-solaractivity (MSA) dataset using the 2011-2013 data, and high-solar-activity (HSA) dataset using the 2014 data. Figure 1 illustrates the solar-activity cycles, and Table 1 presents the frequency of solar flares for each year in the period of our investigation.

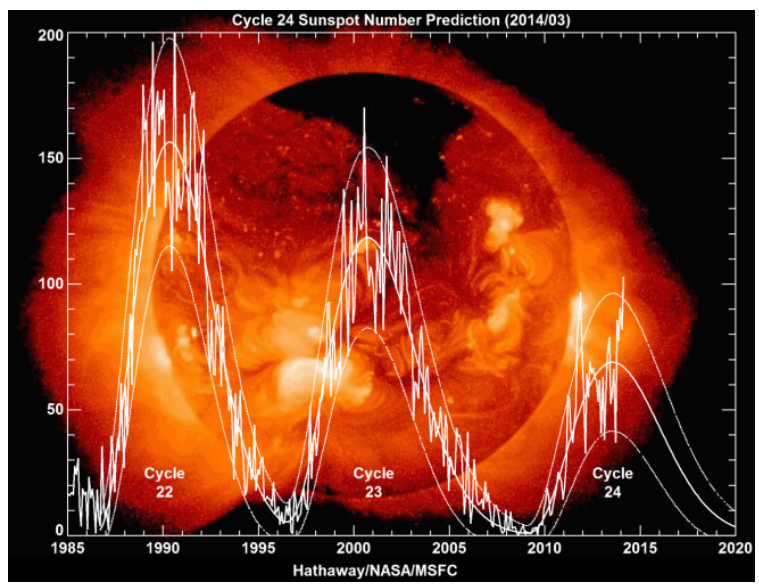

Figure 1. Solar-activity cycle

$\underline{\text { Table 1. Solar flares 2010-2014 }}$

$\begin{array}{ll}2010 & 3 \text { step : } 2 \text { times } \\ 2011 & 3 \text { step : } 10 \text { times } \\ & 4 \text { step : } 2 \text { times } \\ 2012 & 3 \text { step : } 11 \text { times } \\ 2013 & 3 \text { step : } 14 \text { times } \\ 2014 & 3 \text { step : } 17 \text { times }\end{array}$

Although the mean is useful as a numerical representative of an entire dataset, its major limitation is that it loses its representative value if extreme values of the dataset are included in its calculation. In such cases, to identify the data distribution pattern, it is essential to generate a scatter diagram in which the middle position can be identified as a representative value, whereby other information, such as the degree of dispersion of the values scattered around the representative value, can be acquired. There are many methods for measuring the degree of dispersion, such as the deviation and SD. In this paper, the mean and SD, relative $\mathrm{SD}$, and median and quartiles are used in an integrated manner for analyzing the ionosphere. The SD is mathematically expressed as [6]:

$$
\sigma=\sqrt{\frac{\sum_{i=1}^{N}\left(x_{i}-\mu\right)^{2}}{N}}
$$


where $(\mu)$ is the arithmetic mean taken as the data average, $x_{i}$ is an individual data point, $N$ is the number of data points, and $\left(x_{i}-\mu\right)$ is the deviation of each data point from the average [4], [7]. The relative SD (RSD) is the absolute value of the coefficient of variation (CV). It is obtained by dividing the SD by the mean and multiplying the quotient by 100. The RSD is referred to as the CV. Thus, a large RSD, which implies that the SD is relatively large compared with the mean, indicates a large degree of dispersion, and vice versa. Because the RSD has no units, it is a convenient means to compare the dispersion between datasets with different units. The RSD of the foF2 used in this paper is denoted as $V_{R}$ [8]:

$$
V_{R}=\left(\frac{\sigma}{\mu}\right) \cdot 100
$$

As previously mentioned, in cases where extreme values are included in its calculation, the mean loses its usefulness as a representative value, and a scatter diagram should thus be used to estimate the degree of dispersion. In particular, the analysis of a dataset having a symmetrical structure with outliers, such as foF2, is greatly influenced by extremely small or large values. In such cases, because of the mean's low representativeness of the entire sample, order statistics are often used.

Quartiles are obtained by dividing a dataset into quarters containing equal or quasi-equal numbers of data points, whereby the entire dataset (100\%) is cut at $25 \%$, $50 \%$, and $75 \%$ (100\% = entire distribution). If a dataset is arranged in ascending order, the first (Q1), second (Q2), and third (Q3) quartiles correspond to the data points at $25 \%, 50 \%$ (median), and $75 \%$, respectively. The range of the quartiles (IQR) is expressed as follows:

$$
I Q R=Q 3-Q 1
$$
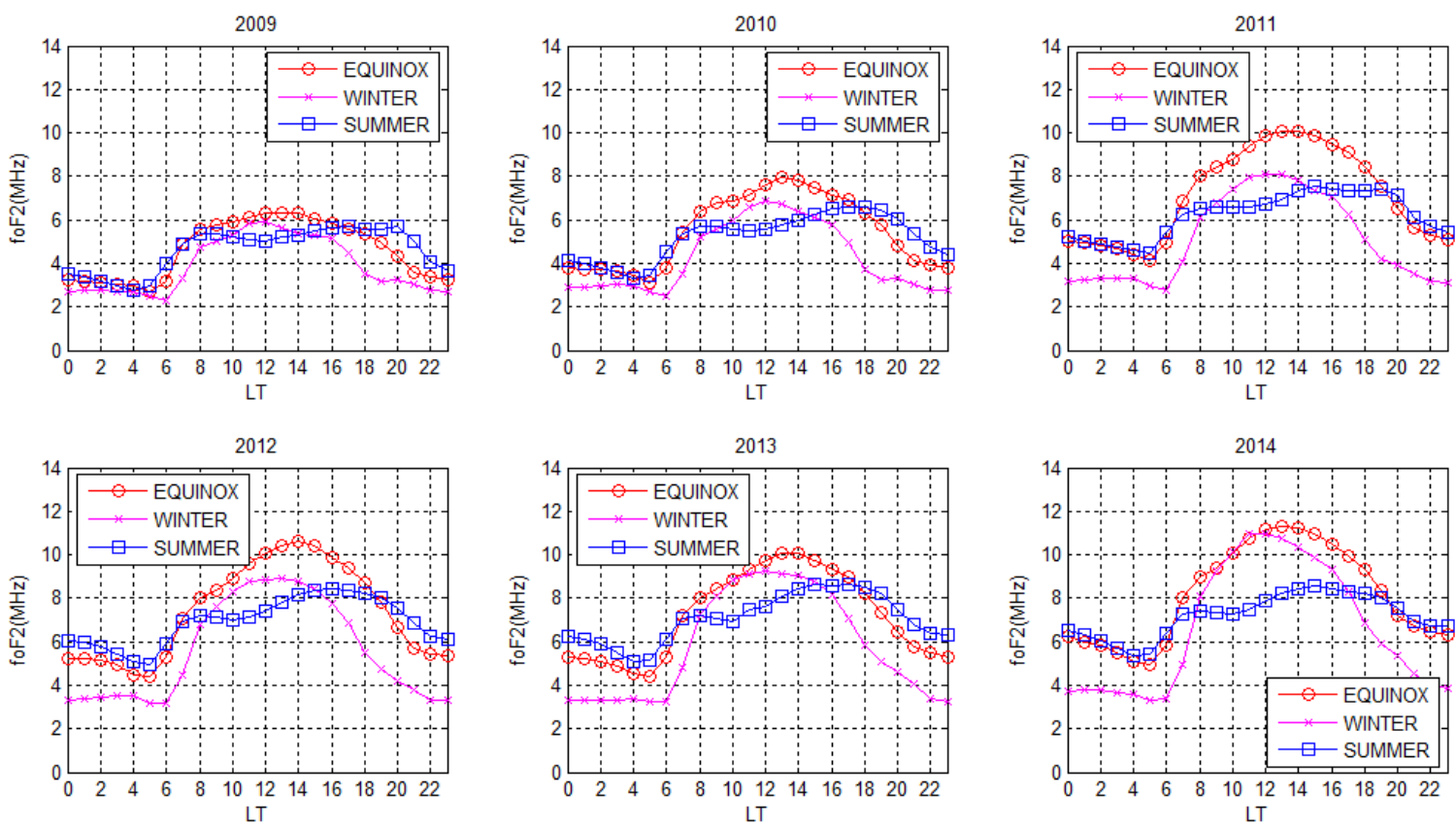

Figure 2. Average foF2 observed in Jeju-do according to solar-aactivity cycles

\section{RESULTS AND DISCUSSIONS}

\subsection{Ionospheric foF2 Observations}

Figure 2 depicts the average variations in the foF2 in Jeju-do throughout the observation period (2009-2014), divided into low-, medium-, and high-solar-activity periods: the LSA (2009 and 2010), MSA (2011-2013), and HSA (2014) periods, respectively. Figure 3 depicts the average variations in the foF2 observed in Icheon-si during the LSA (2010), MSA (2011-2013), and HSA (2014) periods.

In summer, foF2 falls to a trough at 04:00-04:00 LT (local time), gradually rises to a peak at 15:0016:00, and gradually falls from 19:00-20:00 onwards. This reflects the seasonal nature of summer, when 
daylight lasts longer than in other seasons [9]-[11]. In winter, the foF2 falls to a trough at 06:00, gradually rises to a peak at 11:00-12:00, and rapidly falls afterwards. The foF2 is most markedly influenced by solar activity in winter: it exhibits decreased variations at 18:00 under low solar activity and at 22:00 under high solar activity. The variation range becomes large under high solar activity (3.34-11 MHz), as exhibited by the HSA period (2014), increasing by $4.1 \mathrm{MHz}$ compared with the variations during the LSA period (3.6 $\mathrm{MHz}$ ) [12]. This discrepancy is attributed to the location of Korea, which is located near the middle of the northern hemisphere and is susceptible to the influence of solar activity. Comparing the foF2 variation during the LSA (2009) and HSA (2014) periods clearly reveals the reason for the larger foF2 variation according to solar activity in winter. In LSA winter, the foF2 exhibits summer-like variation, whereby the duration in summer is longer than in winter, as a seasonal factor. However, during HSA winter nights (22:00-06:00), the foF2 decreases substantially compared with that in summer, because less solar radiation reaches the Earth, which is tilted against the Sun.
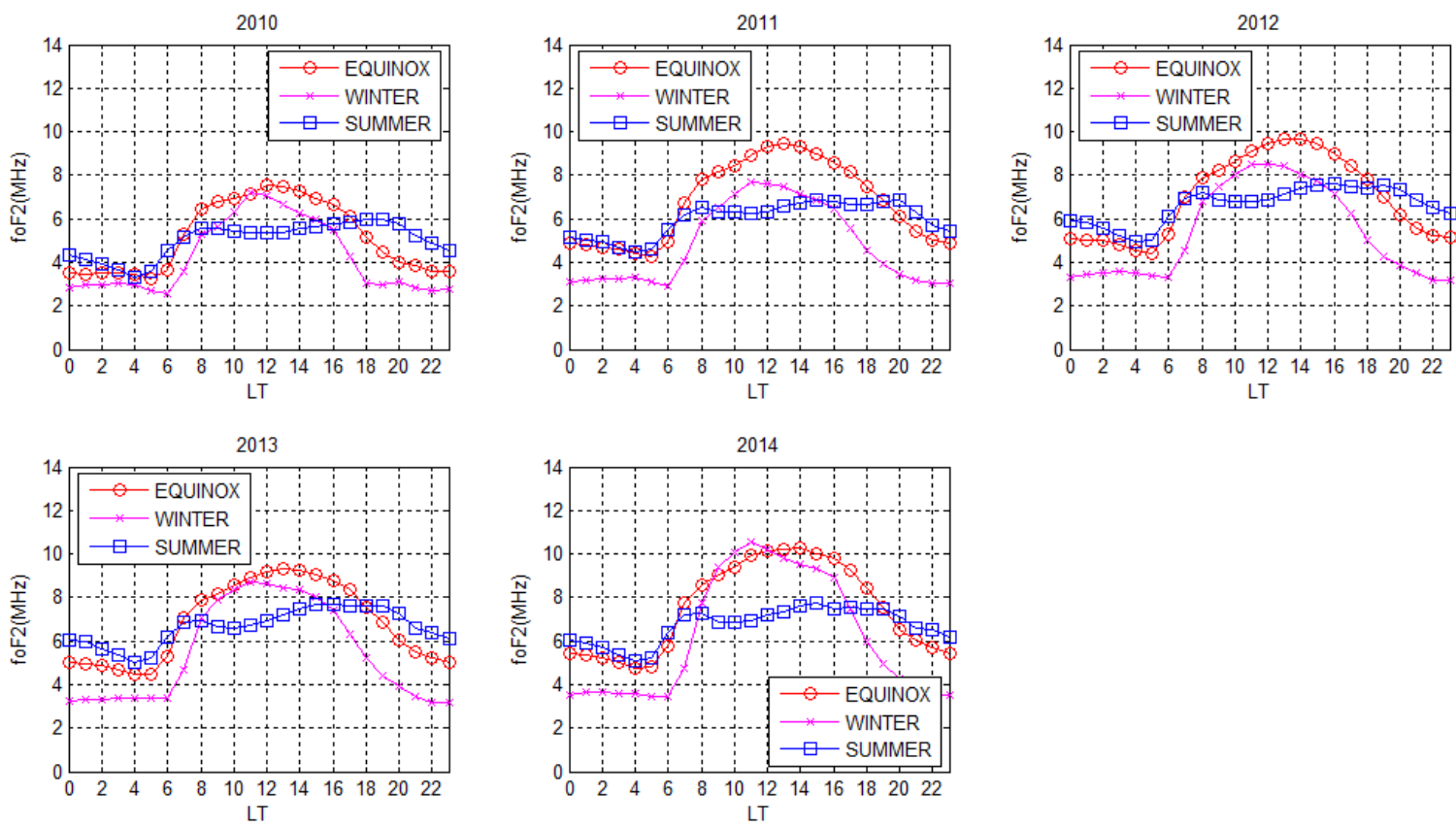

Figure 3. Average foF2 observed in Icheon-si according to solar-activity cycles

During winter days, the foF2 increases to a level far higher than that in summer because of increased solar flares and the lowest meridian altitude, which increases the solar radiation received for the same length of time. This tendency was more remarkable in Icheon-si than in Jeju-do. Such a difference is assumed to become more noticeable at a frequency exceeding 100 sunspot eruptions.

\subsection{Variation of Relative Standard Deviation}

Figures 4 and 5 show the RSDs for the average foF2 values observed in Jeju-do and Icheon-si, respectively, which are shown in Figures 2 and 3.

Figure 4 shows that the RSDs for the average foF2 values in Juju-do were 11-28\% in summer, 9$24 \%$ in winter, and $9-33 \%$ in the equinoxes in the LSA period (2009 and 2010). In the MSA period (20112013), they were $13-21 \%, 9-27 \%$, and $12-30 \%$, respectively, and in the HSA period (2014), they were 1220\%, 14-26\%, and 15-29\%, respectively. Figure 5 shows the same values for Icheon-si. The RSDs in summer, winter, and the equinoxes were $11-18 \%, 9-22 \%$, and 9-33\%, respectively, in the LSA period (2010). In the MSA period (2011-2013), they were 9-25\%, 11-28\%, and 11-29\%, respectively, and in the HSA period (2014), they were 16-25\%, 15-27\%, and 17-32\%, respectively.

Throughout the period of observation, the highest RSDs occurred at dawn (04:00-07:00 LT). This implies that the electron density in the F2 layer varies greatly around dawn because of the increased variations in the ionization due to solar radiation, solar wind, geomagnetic activity, neutral atmosphere, and 
electrodynamics. The low RSD (11-12\% on average at 13:00-15:00 LT) in summer 2014 (HSA) indicates that high solar activity and a long duration yield a small variation in the foF2.
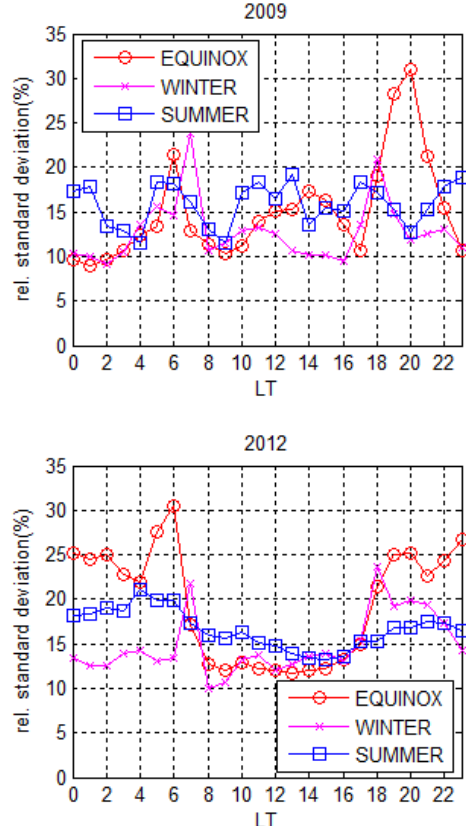
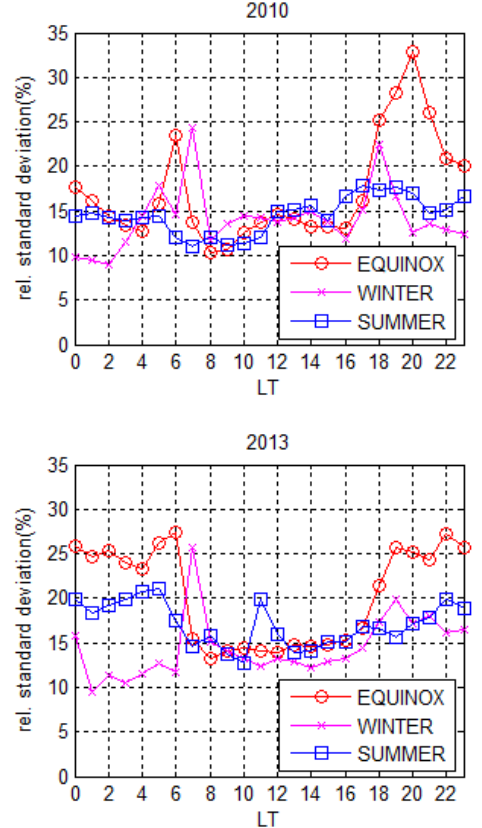
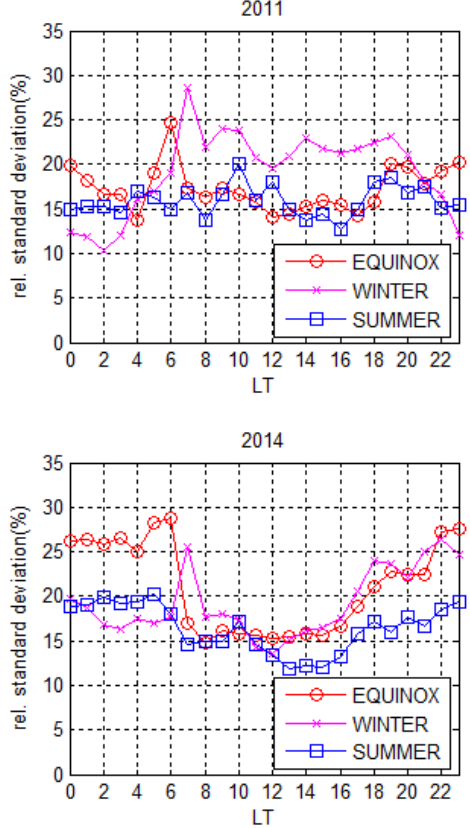

Figure 4. Relative standard deviation of foF2 according to solar-activity cycles in Jeju-do
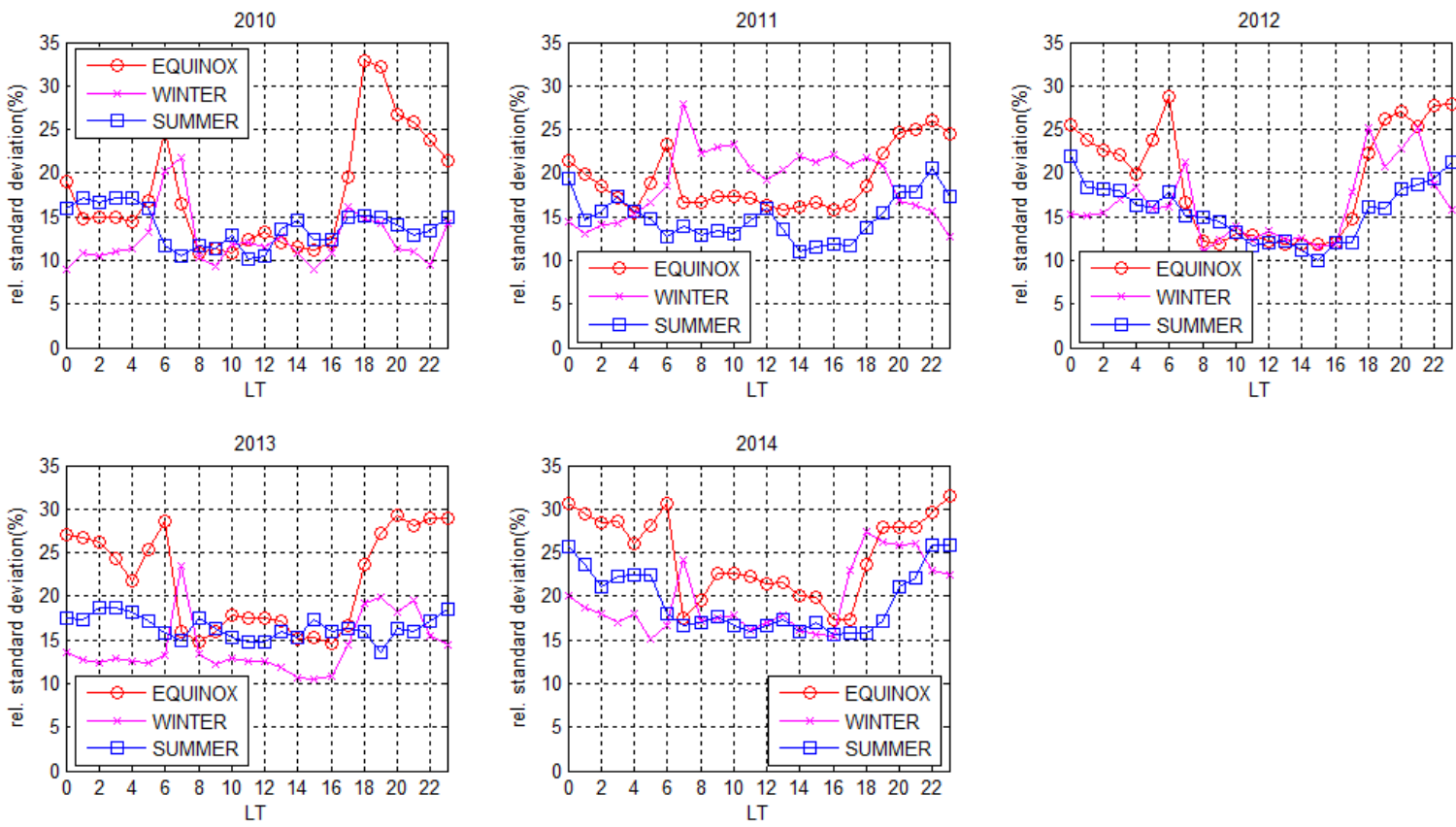

Figure 5. Relative standard deviation of foF2 according to solar-activity cycles in Icheon-si

\subsection{Analysis of foF2 Variations according to Solar-Flare Frequency by Median and Quartiles}

The solar-flare frequencies displayed in Table 1 are closely associated with the solar-activity cycle. Under low solar activity, less than 10 solar flares occurred. The frequency of flares increased with the solar activity. The ionospheric electron density increased in proportion to the frequency of solar flares. 

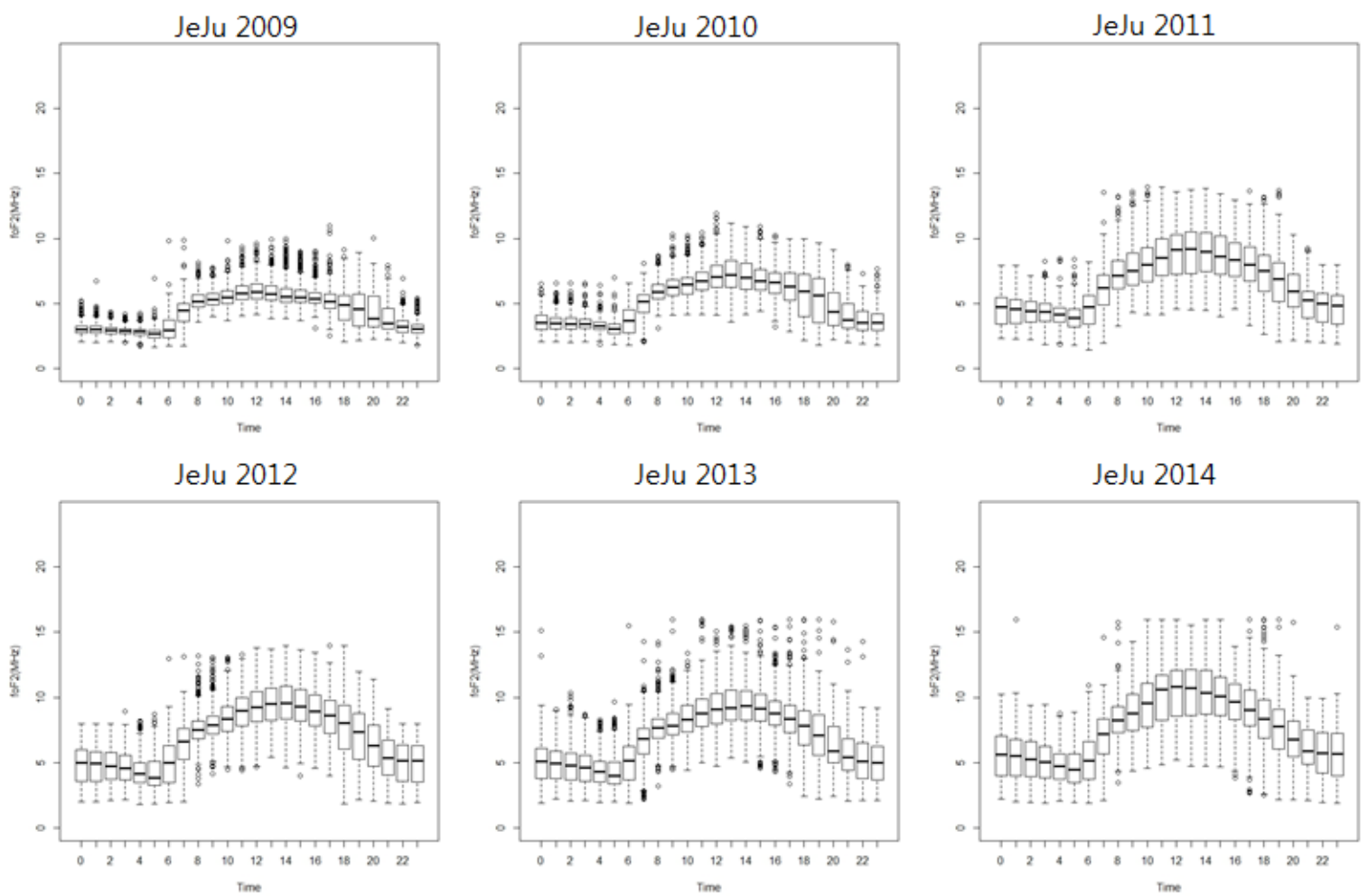

Figure 6. Variations of foF2 in Jeju-do according to solar-flare frequency (median, quartiles, outliers, and whiskers)
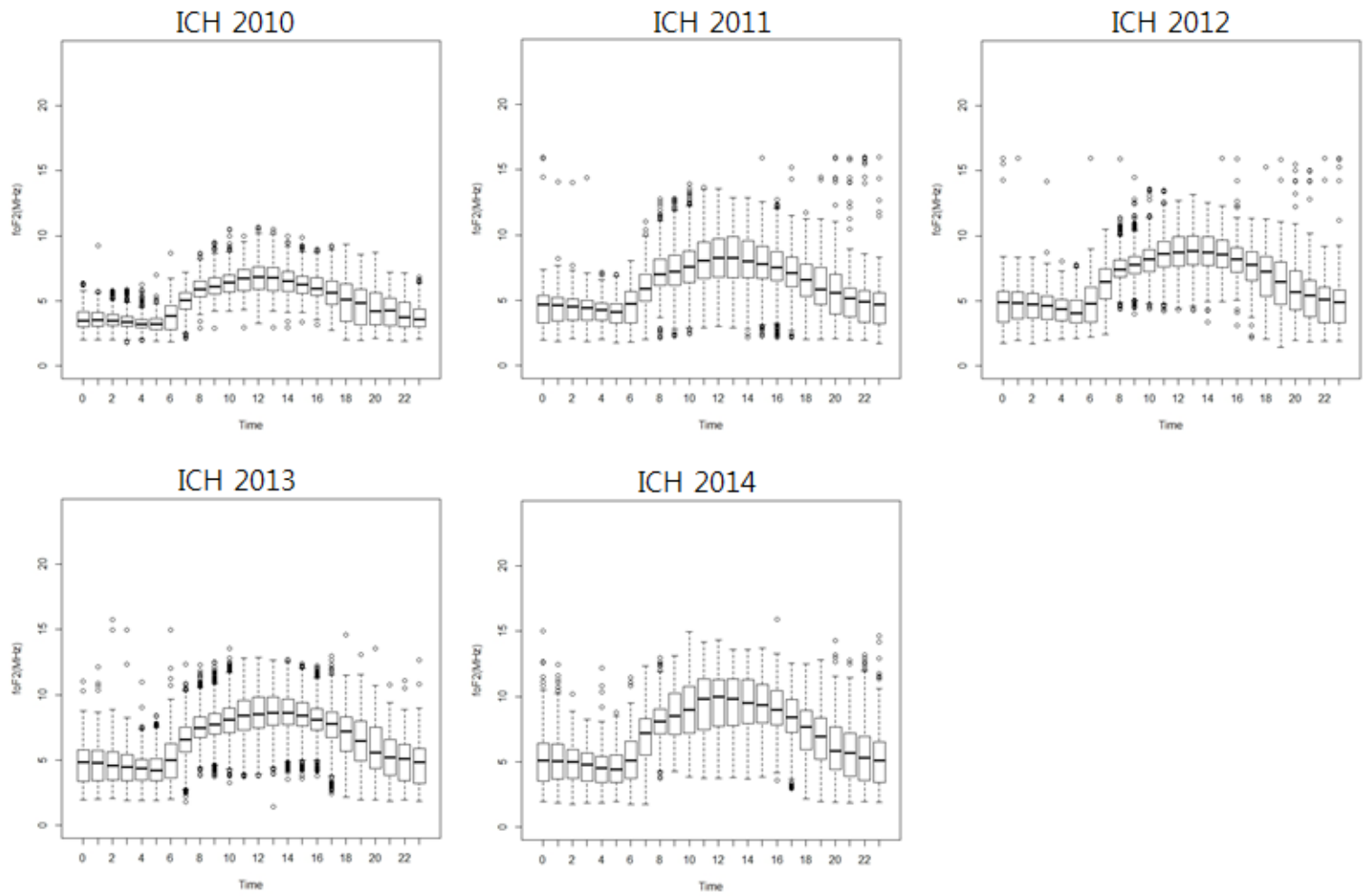

Figure 7. Variations of foF2 in Icheon-si according to solar-flare frequency (median, quartiles, outliers, and whiskers) 
Figures 6 and 7 illustrate the ionospheric electron density according to the solar-flare frequency. The electron-density distributions in the ionosphere were estimated using the median and quartiles. In the graphs of Figures 5 and 6, the thick transverse lines within the boxes represent the median, and the $25^{\text {th }}$ and $75^{\text {th }}$ percentiles are the parts below and above the median line, respectively. That is, the upper 25\% (Q3) and lower 25\% (Q1) from the median comprise the middle 50\%. The vertical dotted lines are referred to as whiskers. For the upper whisker, one of the two values is taken. In the presence of the maximum value or outliers, the largest value is taken from the values larger than the $75^{\text {th }}$ percentile or smaller than the 1.5 interquartile ranges $(1.5 \times \mathrm{IQR})$. The value of $1.5 \times \mathrm{IQR}$ approximates the SD. The IQR is defined as the difference between Q1 and Q3. An outlier is defined as a value falling outside, either the upper or lower $1.5 \times$ IQR boundaries and is expressed as an individual value on the graph. Therefore, in the absence of outliers, whiskers indicate only the highest and lowest values. Moreover, outliers are values that deviate disproportionally from the distribution and are expressed as points on the graph. Outliers include values that are overly extreme, invalid for the dataset, or unrealistic.

Analyses of the ionospheric data for Jeju-do yielded the following findings. In the LSA period (2009 and 2010) with fewer than ten solar flares, a distribution pattern of densely clustered electrons around the median was observed. Since the data for 2009 are incomplete-missing data for the first half of the year-a larger number of outliers were compared with the results of an analysis performed on a complete annual dataset for 2010. Moreover, at 18:00-20:00 LT in 2009 with missing data and in 2010 — an LSA year with fewer than 10 solar flares - the quartiles were found to be widely dispersed around the mean, with outliers rarely occurring. If this result is substituted into the results presented under Section 3.2, it can be assumed that 18:00-20:00 LT is a time slot in which variations in the electron density occur with more intensity than in other time slots throughout the solar-activity cycle.

Analyses of ionospheric data for 2011-2014, in which over 10 solar flares occurred, resulted in the following findings. A large number of outliers were found among the 2012 and 2013 data points, but when the outliers were elevated, similar ionospheric electron-density distribution patterns were confirmed for 2011-2013. In 2014, when solar flares occurred most frequently, outliers occurred less frequently, and the interquartile range was broader than that in other years. This signifies that the ionospheric variations become more intense in cases where solar flares occur over 15 times, owing to the broader distribution of the F2 layer. Icheon-si exhibited similar ionospheric variations, but outliers occurred more frequently. This allows the assumption that the ionospheric variations are predicted more easily in Jeju-do and with more difficulties in Icheon-si owing to frequent outlier occurrences.

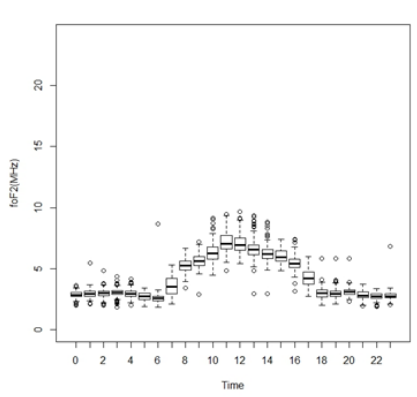

Winter

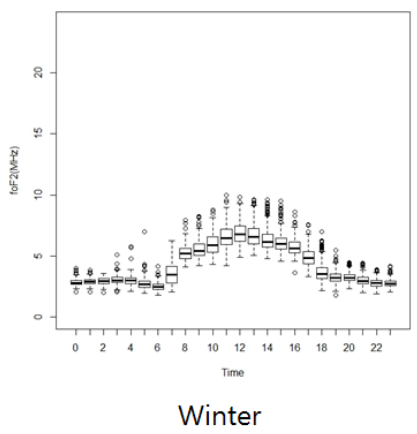

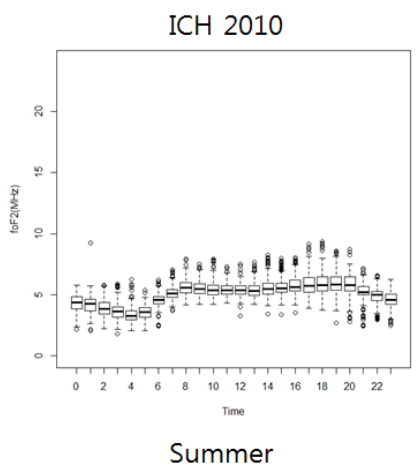

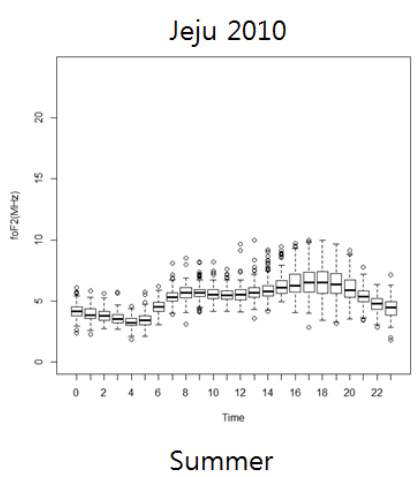

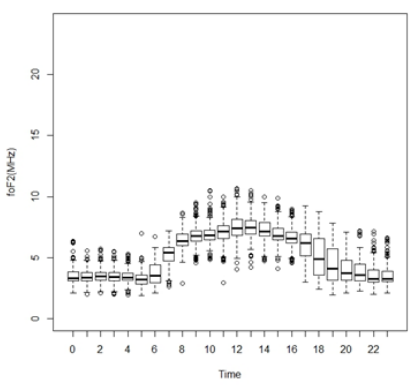

Equinox

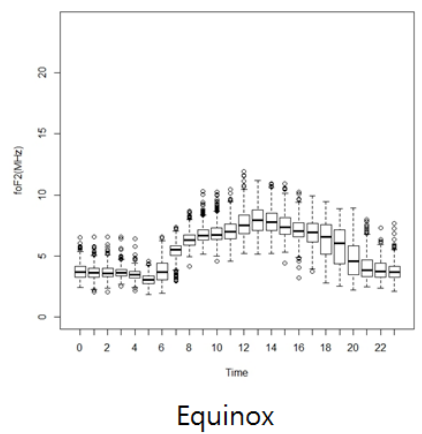

Figure 8. Variations of foF2 in ionosphere above Korea under low solar activity (median, quartiles, outliers, and whiskers) 


\subsection{Analysis of Seasonal foF2 Variations according to Solar Activity by Median and Quartiles}

In order to analyze seasonal foF2 variations according to the solar activity, we derived the median, quartiles, whiskers, and outliers for 2010 (LSA), 2012 (MSA), and 2014 (HSA).

Figure 8 shows the seasonal foF2 variations in 2010 (LSA) observed in Icheon-si and Jeju-do. At both locations, no intense ionospheric variations were observed around the median. Particularly in summer, increased foF2 variations were verified in Icheon-si. This is ascribed to the frequent occurrence of outliers compared with Jeju-do, where less intense foF2 variations were observed owing to the low frequency of outliers. This supports the result derived in Section 3.3 that ionospheric variations can be predicted more easily in Jeju-do than in Icheon-si.

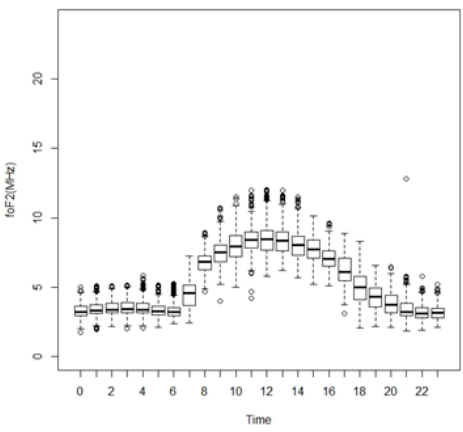

Winter

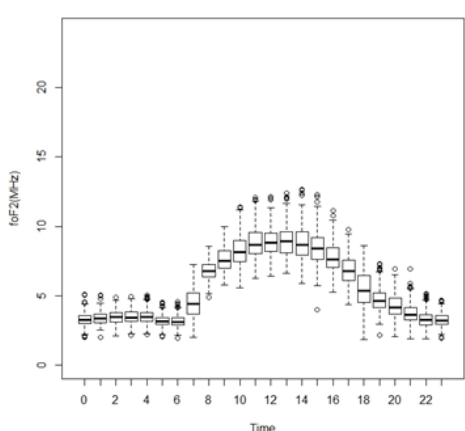

Winter

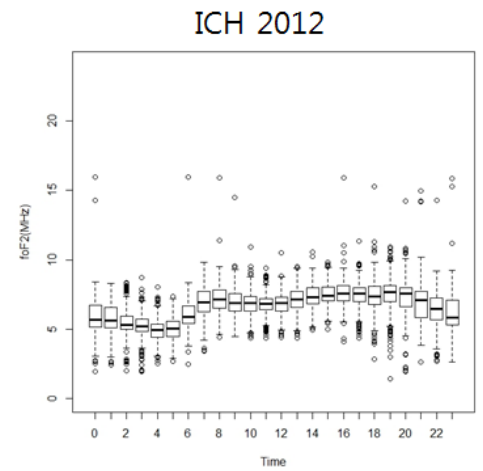

Summer

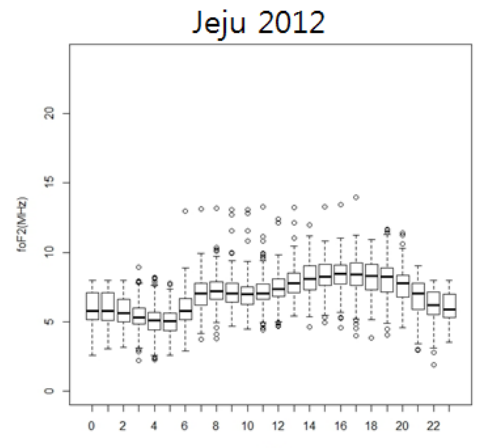

Summer

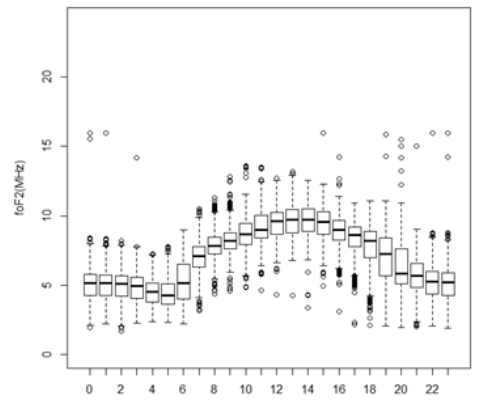

Time

Equinox

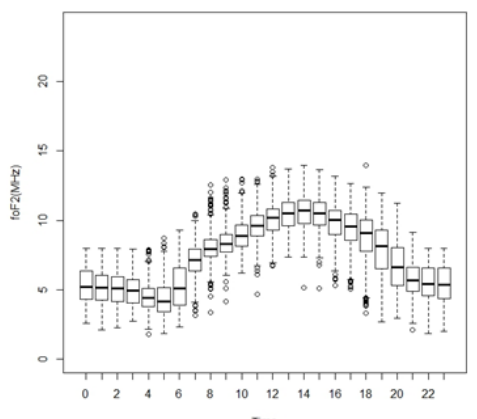

Equinox

Figure 9. Variations of foF2 in ionosphere above Korea under medium solar activity (median, quartiles, outliers, and whiskers)

Figure 9 shows the seasonal foF2 variations in 2012 (MSA). The summer graphs show that the foF2 slightly increased with similar variability to 2010. Similarly, the graphs for the equinoxes and winter show that the overall foF2 increased slightly, with a stronger tendency in winter and equinoxes at 08:00-18:00 and 06:00-20:00, respectively, under low solar activity. The foF2 variations become far more intense at equinoxes compared with the LSA (2010). As in the LSA period (Figure 8), outliers occurred frequently in Isheon-si in the MSA period (Figure 9).

Figure 10 shows the seasonal foF2 variations in 2014 (HSA). Here, compared with the LSA and MSA periods, outliers occurred with a higher frequency. The interquartile ranges were also larger. The distinct feature of analyzing the ionospheric variations using median and quartiles is the identification of the distribution. In summer, while height variations occur over the entire ionosphere, the ionospheric variations have a more consistent distribution compared with those in other seasons. During the analysis of the critical frequencies of the entire ionosphere, gradually increasing tendencies are observed as the solar activity increases, and vice versa. The bar graph in Figure 11 shows the correlations between the critical frequencies at hourly intervals using this feature. The graph shows the 2014 output results for Jeju-do, indicating that very strong time-series correlations exist between the residual series, with decreasing tendency as the time 
interval increases. The analysis of this distinct feature reveals similarities between $f o F 2_{t}$ and $f o F 2_{t-1}$ and using this finding, an unmeasured data point $f o F 2_{N}$ can be included using Eq. (3). If Eq. (3) is employed for predicting ionospheric variations and obtaining missing data for past ionospheric variations using the dataassimilation technique, more reliable analysis results may be derived.

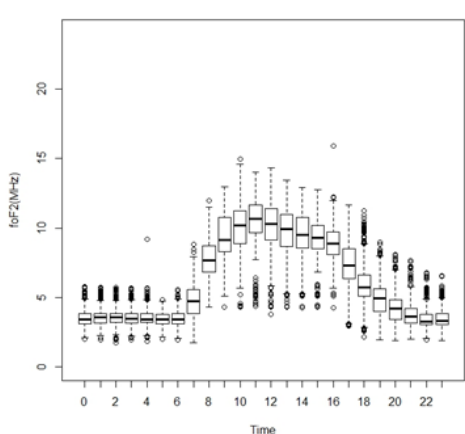

Winter

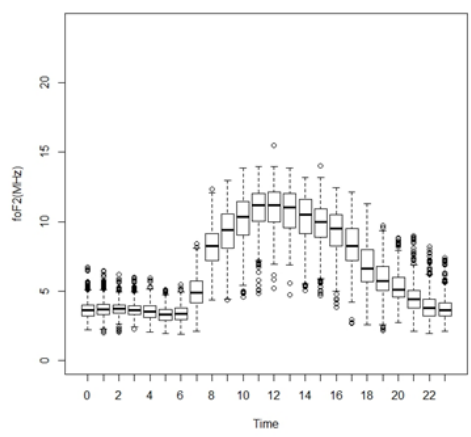

Winter

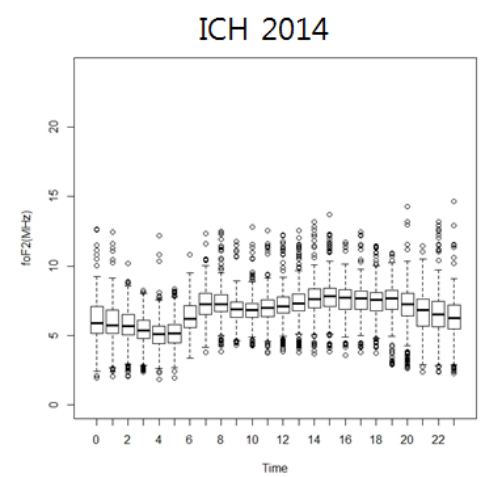

Summer

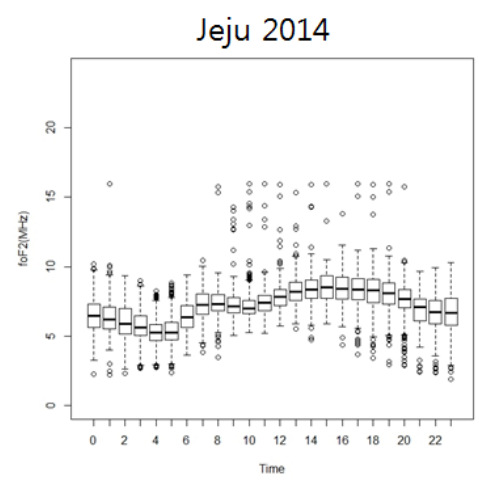

Summer

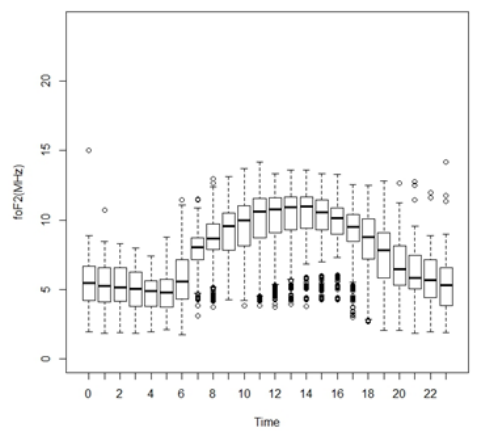

Equinox

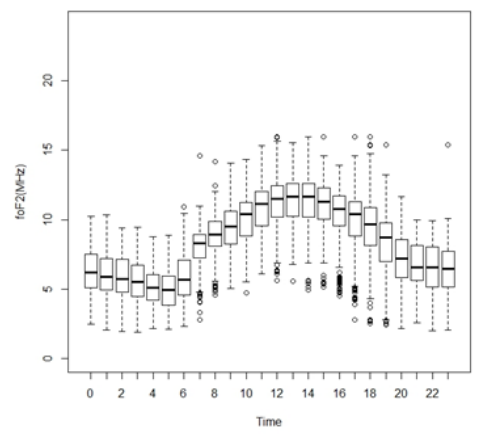

Equinox

Figure 10. Variations of foF2 in ionosphere above Korea under high solar activity (median, quartiles, outliers, and whiskers)

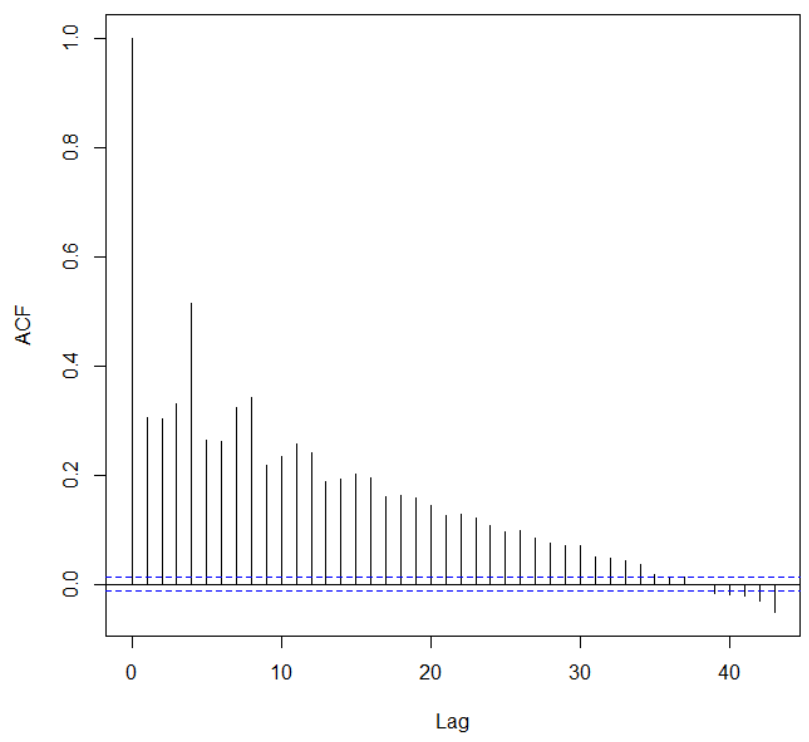

Figure 11. Inter-foF2 correlation graph at hourly intervals 


$$
f o F 2_{N}=\left(f o F 2_{t-2}+f o F 2_{t}\right) / 2(\mathrm{t}-2<\mathrm{N}<\mathrm{t})
$$

\section{CONCLUSION}

The F2 layer is the upper sector of the ionospheric F region, and it is 250 km above sea level. It has a high electron density and thus plays an important role in shortwave communications. Ionospheric variations can be divided into four types: diurnal, seasonal, latitudinal, and solar-cyclic. The critical-frequency (foF2) variations above the Korean Peninsula reach a peak at dawn and fall to a trough at dusk, and the timing for the peak and trough differ according to seasonal variability and solar-activity cycle. We analyzed seasonal and annual foF2 variations and reactions of the F2 layer height at two locations in Korea (Rep.), Jeju-do and Icheon-si by employing the mean and SD, which were used in previous data analyses. Additionally, the median and quartiles were used to describe the variability of the foF2 in the ionospheric analysis. The mean and SD used in previous studies have the advantage of easy analysis of probability but exhibit limitations in developing the SD. The median and quartiles used in the present study can indicate the relative SD and thus describe the range of variations in the foF2 due to solar activity in greater detail. Additionally, measures to enhance the reliability of ionospheric data based on the distinct feature of the range of variations were presented. In a follow-up study, the present study will be extended by using the equation for ensuring the reliability of ionospheric data and comparing the analysis results with the IRI-2012 model.

\section{ACKNOWLEDGEMENTS}

This paper describes the results of research conducted in fulfillment of work commissioned by the 2014 Information and Communication Technology (ICT) and Broadcasting R\&D Program of the Korean Electronics and Telecommunications Research Institute (ETRI), which was funded by the Ministry of Science, ICT, and Future Planning (MSIP).

\section{REFERENCES}

[1] A.D. Danilov, "Variations in foF2 at the end of the 1990s and beginning of the 2000s according to median data", Geomagnetism and Aeronomy, vol. 51, pp. 753-761, dec 2011.

[2] D. Panchevaa and P. Mukhtarov, "A single-station spectral model of the monthly median foF2 and M (3000) F2", Studia Geophysica et Geodaetica, vol. 42, pp 183-196, Apr 1998.

[3] A.D. Danilov and A.V. Konstantinova, "Variations in foF2 at the end of the 1990s and the beginning of the 2000s according to the SPIDR system data", Geomagnetism and Aeronomy, vol. 52, pp. 350-355, May 2012.

[4] D. Bilitza, et al, "Variability of foF2 in the equatorial ionosphere", Advances in Space Research, vol. 34, pp. 19011906, 2004.

[5] M.A. Abdu, et al, "Equatorial ionosphere sunset electrodynamics in the American sector from SUNDIAL December 1988 campaign results", Geomagnetism and Aeronomy, vol. 33, pp. 16-23, Feb 1993.

[6] B.O. Adebesin, et al, "Ionospheric foF2 morphology and response of F2 layer height over Jicamarca during different solar epochs and comparison with IRI-2012 model", Journal of Earth System Science, vol. 123, pp. 751765, Jun 2014.

[7] H. Rishbeth and M. Mendillo, "Patterns of F2-layer variability", Journal of Atmospheric and Solar-Terrestrial Physics, vol. 63, pp. 1661-1680, Oct 2001.

[8] O.A. Oladipo, et al, "Variability of equatorial ionospheric electron density at fixed heights below the F2 peak", Journal of Atmospheric and Solar-Terrestrial Physics, vol. 70, pp. 1056-1065, May 2008.

[9] B.G. Fejer, et al, "Global equatorial ionospheric vertical plasma drifts measured by the AE-E satellite", Journal of Geophysical Research: Space Physics, vol. 100, pp. 5769-5776, Apr 1995.

[10] B.G. Fejer, "The electrodynamics of the low-latitude ionosphere: Recent results and future challenges", Journal of Atmospheric and Solar-Terrestrial Physics, vol. 59, pp. 1465-1482, Sep 1997.

[11] B.O. Adebesin, et al, "Equatorial vertical plasma drift velocities and electron densities inferred from ground-based ionosonde measurements during low solar activity", Journal of Atmospheric and Solar-Terrestrial Physics, vol. 97, pp. 58-64, May 2013.

[12] L. Liu, et al, "Comparative study of the equatorial ionosphere over Jicamarca during recent two solar minima", Journal of Geophysical Research: Space Physics, vol. 117, pp. 1978-2012, Jan 2012. 


\section{BIOGRAPHIES OF AUTHORS}
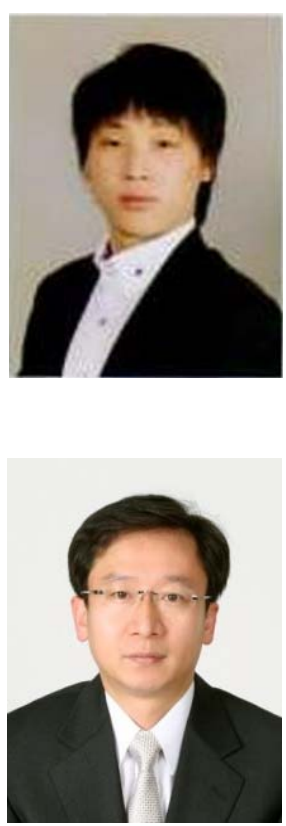

Min-Ho Jeon was born in Jeju, Korea, on Sept. 22, 1984. He received the B.S degree in the department of game digital content from Far East Univ. in 2009, and an M.S. degree in electrical, electronics and communication engineering at the Korea University of Technology and Education in 2011. He is currently pursuing a Ph.D. degree in electrical, electronics and communication engineering at the Korea University of Technology and Education. His research interests are in the context-aware, wireless sensor network, wireless localization, channel coding and M2M network.

Chang-Heon Oh was born in Seoul, Korea, on Feb. 10, 1965. He received the B. S. and M.S.E. degrees in telecommunication and information engineering from Korea Aerospace Univ. in 1988 and 1990, respectively. He received the Ph.D. degree in avionics engineering from Korea Aerospace Univ., in 1996. From Feb. 1990 to Aug. 1993, he was with Hanjin Electronics Co., where he was involved in the research and development of radio communication \& monitoring systems. From Oct. 1993 to Feb. 1999, he was with the CDMA R\&D center of Samsung Electronics Co., where he was involved in the design and development of CDMA cellular systems and CDMA PCS systems for successful commercial CDMA deployment in Korea. Since Mar. 1999, he has been with the School of Electrical, Electronics and Communication Engineering, Korea University of Technology and Education, where he is currently a professor. His research interests are in the areas of wireless communications, mobile communication, and wireless sensor networks with particular emphasis on wireless localization. Dr. Oh is a member of the IEEE Communication Society, IEEE Education Society, Korean Institute of Communication Sciences (KICS), Korea Electromagnetic Engineering Society (KEES), Korea Navigation Institute (KONI), Korea Institute of Information \& Communication Engineering (KIICE), and Korea Institute for Practical Engineering Education (KIPEE). He is currently serving as an Executive Director for the KONI, KIICE and KIPEE. He received the Gold Awards from the Samsung Group in 1998 and the Science Technology Best Paper Award from the Korean Federation of Science and Technology Societies (KOFST) in 2013. 